\title{
A Review on Retinal Feature Segmentation Methodologies for Diabetic Retinopathy
}

\author{
Dr. N. Jayalakshmi ${ }^{1}$, K. Priya ${ }^{2}$ \\ ${ }^{I}$ (Department of Computer Science and Engineering, Saveetha Engineering College, India) \\ ${ }^{2}$ (Research Scholar, Research \& Development Centre, Bharathiar University, India)
}

\begin{abstract}
Diabetic Retinopathy is a most common diabetic eye disease, which occurs when a blood vessel in the retina change. There are two stages of the disease. The early stage is Non proliferative diabetic retinopathy (NPDR) and later is Proliferative diabetic retinopathy (PDR). In NPDR, various problems may occur, such as macular edema which is swelling in the central retina and retinal ischemia which occurs due to poor blood flow. PDR is the advanced stage of NPDR, new blood vessels starts growing in the retina known as neovascularization. The extraction of blood vessels, Optic Disc, Exudates, Macula and Fovea from fundus image of the human retina if done at early stages can be very helpful in diagnosing the severity of the disease and accordingly the treatment can be followed. There are several method present for automatic retinal image feature extraction. In this paper various pre-processing and segmentation techniques are discussed. This review paper describes the different fundus image feature segmentation techniques. The aim of this paper is to review, analyse and categorize the different features of fundus image, giving a brief description, highlighting the key points and the performance measures.
\end{abstract}

Keywords: Blood Vessel, Diabetic Retinopathy, Exudates, Fundus Image, Macula and Fovea, NPDR, Optic Disk, PDR.

\section{Introduction}

The automated disease identification system is not a single process. This system consists of various modules such as (a) Image pre-processing, (b) Anatomical structure identification and feature extraction, (c) Classification techniques, (d) Disease identification. The success rate of each and every step is highly important to ensure the high accuracy of the system. In this paper I have discussed about five methods for Pre-processing and segmentation such as Dharitri Deka et al. [1] the color fundus image is converted into grayscale image and then contrast limited adaptive histogram equalization (CLAHE) [1.13] is applied. First, they detected BV and ROI to segment macula and fovea. T. Ruba et al. [2] they used median filter to remove noisy pixels for preprocessing and gabor feature extraction is applied to detect BV, OD and Exudates. Jyothis Jose et al. [3] proposed median filter for pre-processing to enhance the presence of red lesions. To sharpen the edges of the red lesions, a moat operation is applied and extracted the hard exudates by generating motion patterns. A.V. Deorankar et al. [4] in this paper they focused on HIS means hue saturation and intensity. In this color model space, the intensity component get decoupled from color which carry information in color image hence it is an ideal tool for image processing. Morphological operations are used to detect exudates and micro-aneurysms. Saumitra Kumar Kuri et al. [5] proposed adaptive histogram equalization technique is applied for preprocessing. To segment the vessels under various normal or abnormal conditions they used gabor filter with local entropy thresholding method.

\section{Methods For Fundus Image Pre-Processing}

The aim of Pre-processing is an improvement of the image data that suppresses unwanted distortions or enhances some image features important for further processing. Pre-processing images commonly involves removing low-frequency background noise, normalizing the intensity of the individual particles images, removing reflections, and masking portions of images. 


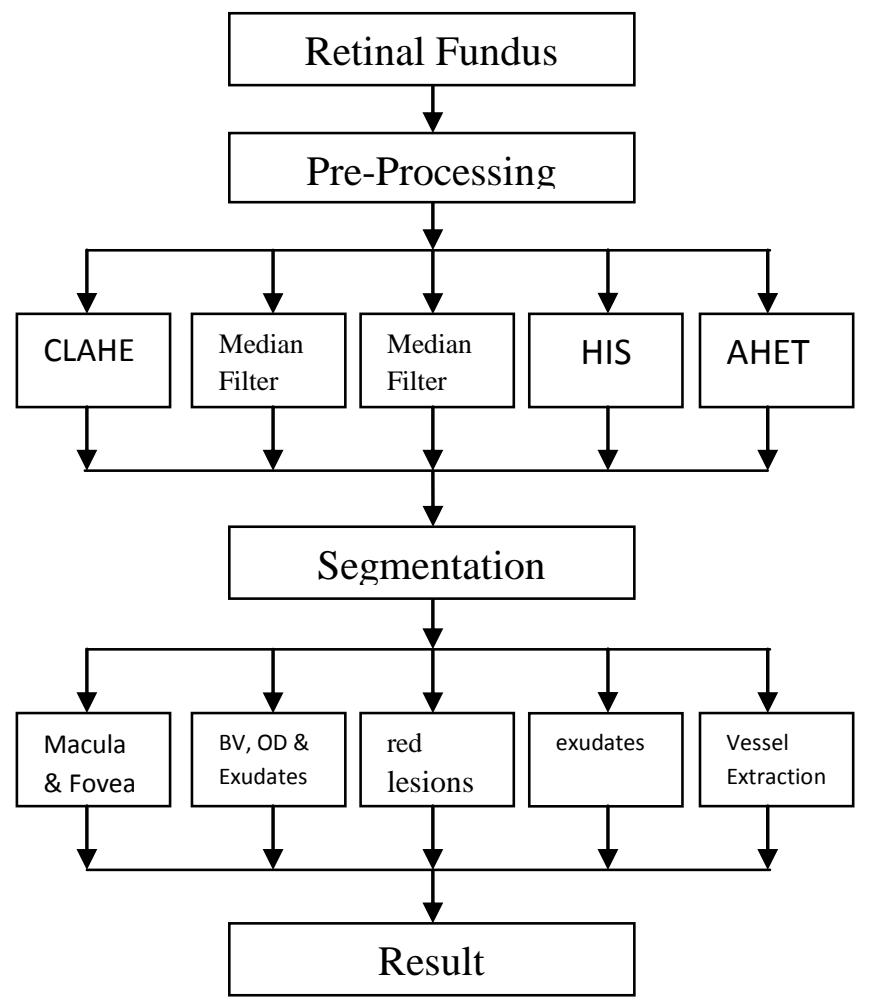

Fig.1.Framework of Literature Survey

All the images are commonly resized to [256 x 256] size. The image is divided into small regions. Median filter identifies the noisy pixels within the regions and replace the noisy pixels with the median of the neighbouring pixels within the regions. The RGB component of the color input image was separated and preprocessed. Then pre-processed RGB components were concatenated to obtain the filtered image. Fig 3 shows the original fundus image and the pre-processed image.

(a)

(b)
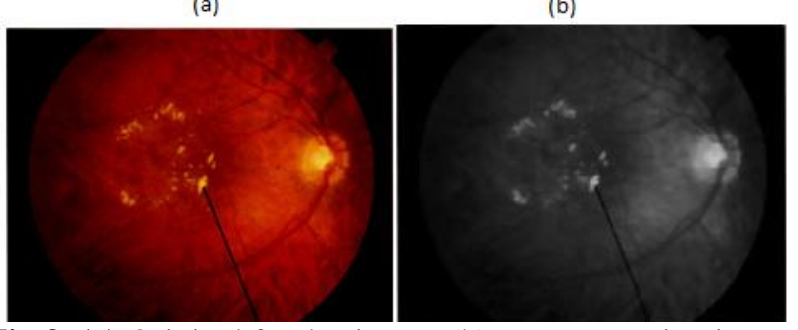

Fig.3. (a) Original fundus image (b) Pre-Processing image

To segment red lesions, the intensity information of both green and red channels of the same fundus image is used for detecting the red lesions. To acquire this histogram matching is used in which the histogram of the green component of the image is modified with the histogram of the red component of the same retinal image to obtain a new image contain advantages of both red and green channels Fig 4 shows the Pre-processed fundus image.

(a)

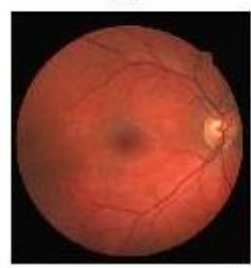

(b)

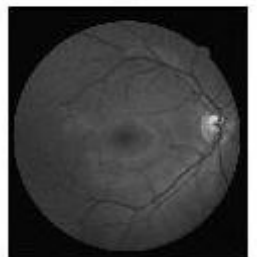

(c)

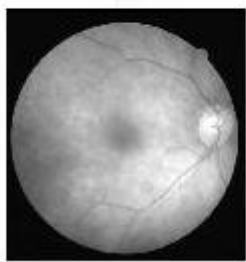

Fig.4.a) Color fundus image (b) Green channel image (c) Pre-Processing image

In this proposed method, the image is get rectified from the problems such as blurring, non-clarity and size. The transformation equations used in the conversion of RGB to HSI. The converted images are then 
filtered to remove noise using hybrid median filter. The CLACHE means the contrast limited adaptive histogram equalization is performed after filtering for contrast enhancement, which improves the quality of images.

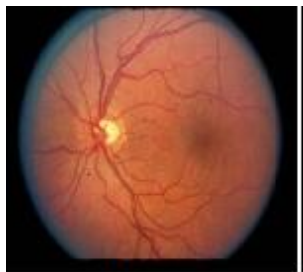

(a)

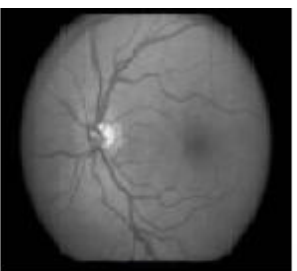

(b)

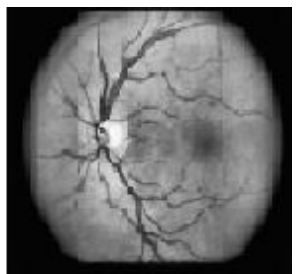

(c)

Fig.5. (a) Original fundus image (b) HIS model image (c) Pre-Processing image

Pre-processing is used to enhance the contrast in the fundus. Low contrast that cause to hardly segment the blood vessels in retinal image. Green channel has highest contrast compare to other channel [6]. Adaptive histogram equalization technique is applied for improving the contrast of the green channel retinal image.

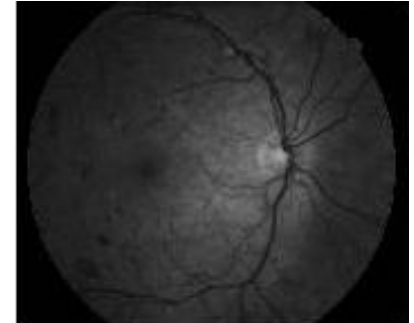

(a)

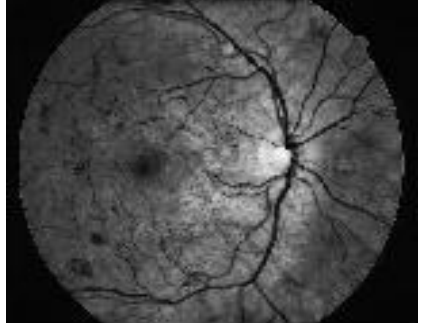

(b)

Fig.6. (a) Green channel of the original image (b) Adaptive histogram equalization image

\section{Various Segmentation Methods For Pre-Processed Images}

Image segmentation is the process of subdividing a digital image into multiple segments (sets of pixels, also known as super pixels). Segmentation is a process of grouping together pixels that have similar attributes [7]. Segmentation can be classified as follows: Region Based, Edge Based, Threshold, Feature Based Clustering, and Model Based [8].

\subsection{Detection of Blood Vessels}

In this proposed method, for detection of blood vessel $5^{\text {th }}$ order discrete wavelet transform (DWT) decomposition and morphological opening operation is applied. After applying $5^{\text {th }}$ order DWT on the preprocessed image the resultant retinal images comprises of the approximated background. Later morphological opening is applied on the image using a ball structuring element of appropriate radius so that BV components are fully suppressed. The resultant image is inverted and subtracted from inverted pre-processed image. Finally the resultant image consists of BV. For removal of circular boundary canny edge detection is applied and morphological filling operation is done. Then the boundary is determined using the following formula $\mathbf{b}=\mathbf{D}^{\mathrm{B}}(\mathbf{I})-\mathbf{E}^{\mathrm{B}}(\mathbf{I})$

Where $b$ is the fundus boundary, $D^{B}(I)$ represents the dilation of image $I, E^{B}(I)$ represents the erosion of the structuring element. Finally resultant image contains BV.

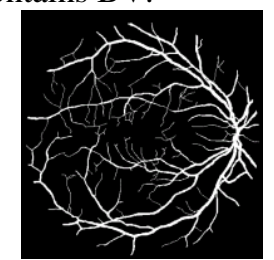

Fig.7.Segmented Blood Vessel

\subsection{Feature Extraction}

The features were extracted using Gabor and GLCM feature extraction methods. These methods give the information about texture of the image based on shapes of the objects in the images. Gabor filter is a direct filter used for edge detection.in this proposed method they were extracting 24 features which includes mean, variance and standard deviation at different orientation by using gabor filter. A gray level co-occurrence matrix 
(GLCM) contains information about the position of pixels having similar gray level values. They were extracting 12 different statistical features like dissimilarity, contrast, correlation, cluster, prominence, cluster shade, energy, entropy, homogeneity, and maximum probability, sum of square, auto correlation, and inverse different moment.

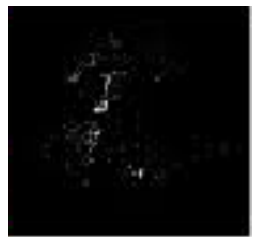

Fig.8.Segmentation of exudates

\subsection{Detection of Retinal Structures}

Morphological image processing [9], [10] is used to determine the retinal blood vessels from the color fundus image. Adaptive equalization is performed on green channel of the input color fundus image. Gaussian filter of size 10 and standard deviation 3.28 is applied on the image. The image is converted to binary image by applying suitable threshold value. Median filtering is performed to remove the noise [9]. Fovea is detected based

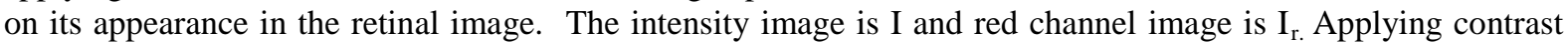
enhancement to both images to obtain $I_{a}$ and $I_{b}$ respectively. Then calculate $I s=I_{a}-\left(I_{b}\right)^{c}$. Binarize by applying a global threshold to detect the fovea [11]. Optic disc is the brightest retinal structure. The green channel of the color fundus image is extracted and converted to binary image by applying a high threshold value. Segment with maximum area on the binary image which will be the one corresponding to optic disc then it shows OD [11], [12].

(a)

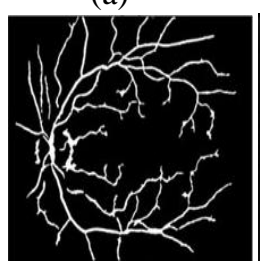

Fig.9. (a) Segmentation of BV (b)

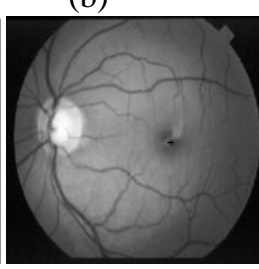

(c)

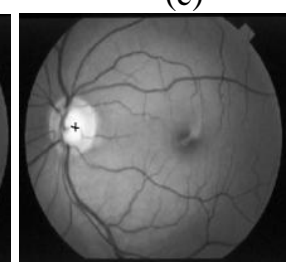

(b) Detection of Fovea (c) Detection of Optic Disk

\subsection{Detection of Micro-aneurysms and Exudates}

The Micro-aneurysms were extracted in five steps are candidate extraction, Optical disc elimination, Blood vessel extraction and removal, Exudates identification and Detection of micro-aneurysms. In candidate extraction Morphological operations such as erosion, dilation, closing, and opening are performed for finding micro-aneurysms. Then the image is inverted. Canny edge detection is used for counter detection [13]. The canny edge detects the edges where intensity of image is getting changes. Canny edge detection algorithm preserve all local maxima the gradient for enhances blurred edges, through this it detects optimal boundaries of features. For detection of exudate remove blood vessel and optical disc is must from retinal image. Structure elements (SE) are in different shapes like diamond, disc, round, rectangle etc. if SE starts from bright pixel, there will be no change and it move to next pixel to next pixel.But if SE dilation which adds pixels to pixels and create boundaries of objects in an image. To remove pixels on object boundaries erosion method is used. Exudates are detected by using morphological operation closing. This closing operation applied on eroded image. In closing dilation is followed by erosion operator. So it detects the exudates. For micro-aneurysms detection opening operation is performed in which erosion is followed by dilation whereas the micro-aneurysms are appearing as red spot which get swell in the retina. Invert image method is used and then morphological opening operation is performed for the detection of micro-aneurysms.

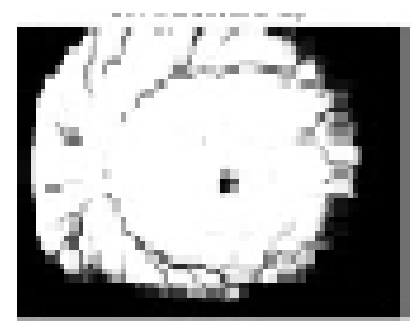

Fig.10.Micro-aneurysms Detection Image 


\subsection{Texture extraction using Gabor filter}

Gabor filter has been applied to texture extraction. Sinusoids modulated Gabor filter kernels are used in this analysis that detected the blood vessels. The gray level co-occurrence matrix (GLCM) contains the information of gray-level transitions in an image [14]. A gabor filter response image has a size of $\mathrm{M} * \mathrm{~N}$ with $\mathrm{L}$ gray levels that converted co-occurrence matrix of this image is an $\mathrm{L} * \mathrm{~L}$ square matrix. The probability of cooccurrence $t_{\mathrm{ij}}$ of gray levels $I$ and $\mathrm{j}$ is normalizing the probability within individual quadrants. The sum of probabilities of each quadrant equals to one, get the cell probability [15].

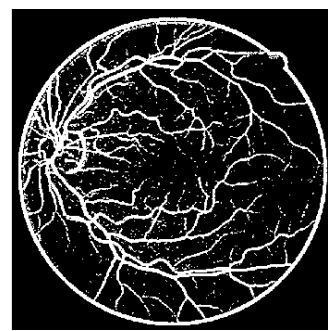

Fig.11.Segmented image

\section{Result And Discussion}

A detection of different features of eye image by different authors are implemented using MATLAB and results are shown in the pictures. And also obtained results are discussed by means of performance measures shown in Table 1.

Table 1: Segmentation of Retinal Features

\begin{tabular}{|c|c|c|c|c|c|}
\hline S.No. & Authors & Features & $\begin{array}{l}\text { Pre-Processing } \\
\text { Methods }\end{array}$ & Segmentation Methods & $\begin{array}{l}\text { Performance } \\
\text { measure }\end{array}$ \\
\hline 1 & $\begin{array}{l}\text { Dharitri Deka et } \\
\text { al. }\end{array}$ & $\begin{array}{l}\text { Blood vessel } \\
\text { structure(Macula } \\
\text { and Fovea) }\end{array}$ & $\begin{array}{l}\text { Contrast limited } \\
\text { adaptive histogram } \\
\text { equalization } \\
\text { (CLAHE). }\end{array}$ & $\begin{array}{l}\text { 5th order Discrete Wavelet } \\
\text { Transform decomposition } \\
\text { and Morphological opening } \\
\text { operation. }\end{array}$ & Accuracy $97.85 \%$ \\
\hline 2 & T.Ruba et al. & Exudates & $\begin{array}{l}\text { Median } \quad \text { Filtering } \\
\text { process. }\end{array}$ & $\begin{array}{l}\text { Morphological operation is } \\
\text { used after thresholding to } \\
\text { separate exudates. }\end{array}$ & Accuracy $99.35 \%$ \\
\hline 3 & $\begin{array}{l}\text { Jyothis Jose el } \\
\text { al. }\end{array}$ & $\begin{array}{lr}\text { Blood } & \text { Vessel, } \\
\text { Optic } & \text { Disc, } \\
\text { Fovea } & \end{array}$ & $\begin{array}{l}\text { Histogram of the } \\
\text { green and red } \\
\text { component are used. }\end{array}$ & $\begin{array}{l}\text { Discrete fourier transform } \\
\text { and high pass filtering. }\end{array}$ & Accuracy $92 \%$ \\
\hline 4 & $\begin{array}{l}\text { JayakumarLach } \\
\text { ure el al. }\end{array}$ & $\begin{array}{l}\text { Micro-aneurysms } \\
\text { and Exudates }\end{array}$ & $\begin{array}{l}\text { Hue saturation and } \\
\text { intensity model }\end{array}$ & $\begin{array}{ll}\begin{array}{l}\text { Morphological opening } \\
\text { operation }\end{array} & \\
\end{array}$ & $\begin{array}{l}\text { Specificity } 100 \% \\
\text { and sensitivity } 90 \%\end{array}$ \\
\hline 5 & $\begin{array}{l}\text { Saumitra } \\
\text { Kumar Kuri }\end{array}$ & Blood Vessel & $\begin{array}{l}\text { Adaptive Histogram } \\
\text { Equalization } \\
\text { technique }\end{array}$ & $\begin{array}{l}\text { Gabor filter and local } \\
\text { entropy threshold }\end{array}$ & $\begin{array}{lr}\text { Accuracy } & 97.72 \% \\
\text { and } & \text { Sensitivity } \\
98.15 \% & \end{array}$ \\
\hline
\end{tabular}

\section{Conclusion}

Automatic methods for the detection of retinal structures such as blood vessels, macula and fovea, optic disk, exudates etc in the color fundus images are discussed with algorithm. Future work based on the identification of different classifiers for classifying segmented retinal images into normal or abnormal image.

\section{References}

[1]. Dharitri Deka, Jyoti Prakash Medhi, S. R. Nirmala, "Detection of macula and fovea for disease analysis in color fundus images," in IEEE $2^{\text {nd }}$ International Conference on Recent Trends in Information System, 2015

[2]. T. Ruba, K. Ramalakshmi, "Identification and Segmentation of exudates using SVM classifier," IEEE Sponsored $2^{\text {nd }}$ International Conference on Innovation Embedded and Communication Systems, 2015

[3]. Jyothis Jose, Jinsa Kuruvilla, "Detection of red lesions and hard exudates in color fundus images," Internation Journal of Engineering and Computer Science ISSN: 2319-7242 Volume 3 Issue 10 October, 2014 page No. 8583-8588

[4]. JayakumarLachure, A. V. Deorankar, Sagar Lachure, Swati Gupta, Romit Jadhav, "Diabetic retinopathy using morphological operations and machine learning," IEEE International Advance Computing Conference, 2015

[5]. Sawmitra Kumar Kuri, "automatic diabetic retinopathy detection using gabor filter with local entropy thresholding," IEEE $2^{\text {nd }}$ International Conference on Recent Trends in Information System, 2015

[6]. P. C. Siddalingaswamy, K. Gopalakrishna Prabhu, "Automatic Segmentation of Blood Vessels in Colour Retinal Images using Spatial Gabor Filter and Multiscale Analysis," $13^{\text {th }}$ International Conference on Biomedical Engineering, IFMBE Proceedings Volume 23, 2009, pp 274-276 SpringerEfford, 2000

[7]. Segmentation Techniques Comparison in Image Processing R. Yogamangalam et al. / International Journal of Engineering and Technology (IJET) ISSN: 0975-4024 Vol 5 No 1 Feb-Mar 2013

[8]. U R Acharya, C M Lim, E Y K Ng, C Chee and T Tamura "Computer based detection of diabetes retinopathy stages using digital fundus images" Proceedings of the institution of Mechanical Engineers, Part H: Journal of Engineering in Medicine 2009 223: 545 
[9]. Thomas Walter_, Jean-Claude Klein, Pascale Massin, and Ali Erginay, "A Contribution of Image Processing to the Diagnosis of Diabetic Retinopathy-Detection of Exudates in Color Fundus Image of the Human Retina" IEEE Transactions on Medical Imaging, Vol. 21, No. 10, October 2002

[10]. J. Singh, G. D. Joshi, and J. Sivaswamy, "Appearance based object detection in color retinal images, "in Proc. Int. Conf. Image Process., 2008, pp. 1432-1435.

[11]. Angel Suero, Diego Marin, Manuel E. Gegundez-Arias, And Jose M. Bravo: Locating The Optic Disc In Retinal Images Using Morphological Techniques", IWBBIO 2013. Proceedings Granada, 18-20 March, 2013

[12]. Li Tang, Meindert Niemeijer, Joseph M. Reinhardt, Mona K. Garvin, and Michael D. Abramoff, "Splat Feature Classification with Application to Retinal Hemorrhage Detection in fundus Images', IEEE Trans Med Imaging. 2013 Feb; 32(2):364-75. Doi: 10.1109/TMI.2012.2227119. Epub 2012 Nov [14] R. M. haralick, K. Shanmugan, I. Dinstein, "Textural Features for Images Classification," IEEE Trans. System, Man and Cybernetics. Vol. SMC-3, No - 6, Nov 1973, 610-621

[13]. Pun, T., 'A new method for gray-level picture thresholding using the entropy of the histogram', Signal Process, 1980, 2, pp. 223 237. 